\title{
Author Correction: Variational quantum unsampling on a quantum photonic processor
}

Jacques Carolan (i), Masoud Mohseni, Jonathan P. Olson, Mihika Prabhu, Changchen Chen, Darius Bunandar (D), Murphy Yuezhen Niu, Nicholas C. Harris, Franco N. C. Wong, Michael Hochberg, Seth Lloyd and Dirk Englund (1)

Correction to: Nature Physics https://doi.org/10.1038/s41567-019-0747-6, published online 13 January 2020.

In the version of this Article originally published online, in the Acknowledgements, the support from the MITRE Quantum Moonshot Program was mistakenly not mentioned; this information has now been added to the sentence beginning "This work was supported by...". All versions of the Article have been amended.

Published online: 4 February 2020

https://doi.org/10.1038/s41567-020-0818-8

(c) The Author(s), under exclusive licence to Springer Nature Limited 2020

\section{Author Correction: Chip-to-chip quantum teleportation and multi-photon entanglement in silicon}

Daniel Llewellyn, Yunhong Ding (iD, Imad I. Faruque (D), Stefano Paesani (D), Davide Bacco (iD, Raffaele Santagati (D), Yan-Jun Qian, Yan LiD, Yun-Feng Xiao Di, Marcus Huber (D), Mehul Malik D, Gary F. Sinclair, Xiaoqi Zhou, Karsten Rottwitt, Jeremy L. O'Brien, John G. Rarity, Qihuang Gong, Leif K. Oxenlowe (D), Jianwei Wang (iD and Mark G. Thompson (iD

Correction to: Nature Physics https://doi.org/10.1038/s41567-019-0727-x, published online 23 December 2019.

In the version of this Letter originally published, the following sentence was missing from the Acknowledgements: "D.L., I.I.F., J.G.R. and M.G.T. acknowledge support from UK Quantum Technology Hub for Quantum Communication Technologies funded by EPSRC: EP/M013472/1; programme grant no. EP/L024020/1." It has now been added in the online versions of this Letter.

Published online: 24 February 2020

https://doi.org/10.1038/s41567-020-0840-x

(c) The Author(s), under exclusive licence to Springer Nature Limited 2020 